\title{
Husten, Schnupfen, Fieber - was Ihre Patienten nun wissen wollen
}

W enn das Wetter Kapriolen schlägt, macht so manches Immunsystem schlapp. Dann drängen sich in Ihrer Praxis wieder Patienten, die wissen wollen, wie sie sich besser schützen und ihre Beschwerden lindern können. In einer Umfrage haben uns Allgemein- und Kinderärzte die häufigsten Fragen genannt, die Eltern und Patienten in diesem Zusamenhang stellen. Daraus ist nun eine Broschüre entstanden, die diese Fragen beantwortet.

Die Patientenbroschüre „20 Fragen 20 Antworten“ zum Thema „Erkältung und fieberhafter Infekt" erklärt dabei nicht nur, ab wann eine erhöhte Körpertemperatur wirklich als Fieber bezeichnet wird und bei welchen Komplikationen besser der Arzt zu Rate gezogen werden sollte. Auch die Frage „Was unterscheidet einen grippalen Infekt von einer richtigen Grippe?" wird in der 28-seitigen Broschüre beantwortet.

Durch die Broschüre sollen die wichtigsten Probleme ausführlich dargestellt und beantwortet werden, sodass in der Sprechstunde mehr Zeit für gezieltere Fragen bleibt. Daneben wird die Arztempfehlung zur Selbstmedikation durch das Grüne Rezept unterstützt.

EEG von Ingmar Wellach eminent wichtig für die Erlangung profunder Kenntnisse.

Das neu erstellte Praxisbuch ist aus einem Skript für die Weiterbildungsassistenten hervorgegangen. Im allgemeinen Teil werden die Grundlagen ausführlich genug beschrieben, während im speziellen Teil die allermeisten normalen und pathologischen Befunde und Veränderungen dargestellt werden.

Hier zeichnet sich das vorliegende Buch mit seinen übersichtlichen Darstellungen und der präzisen Kurvendiskussion aus. Es ist auch für Anfänger verständlich geschrieben, bietet umfassen-

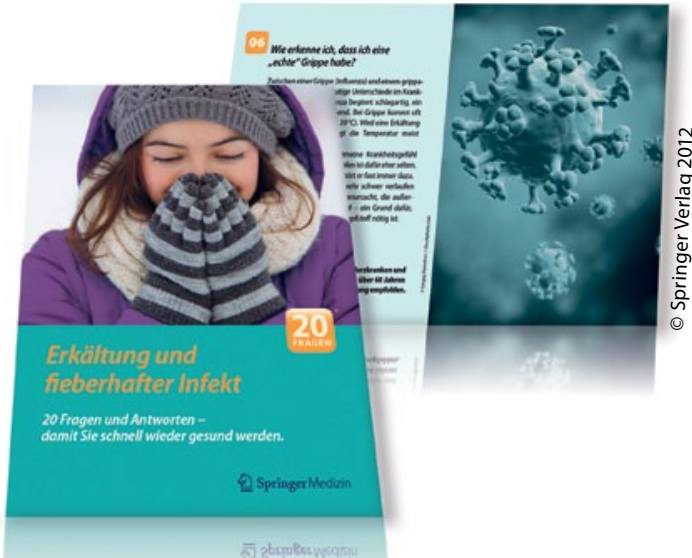

Die Broschüre kann von allen Ärzten (immer im Paket von 100 Exemplaren) fürs Wartezimmer bestellt werden. Per E-Mail an: vertrieb@springer.com oder per Fax an: 061 02/50 62 40, Stichwort „Erkältung und fieberhafter Infekt“. red de Informationen und ist im klinischen Alltag sehr nützlich. Insgesamt ist das Werk für alle Neurologen und Neuropädiater sehr empfehlenswert und vermittelt $\mathrm{zu}$ einem fairen Preis alle erforderlichen Kenntnisse für das EEG-Zertifikat.

Dr. F. Grüttner

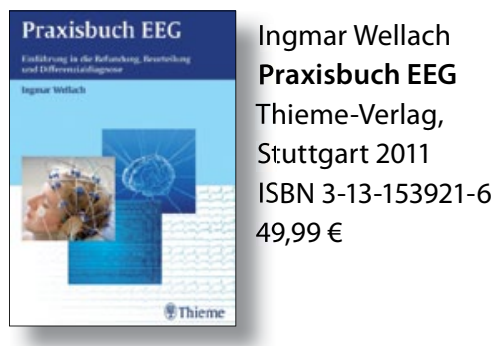

\section{Junge Wilde für die Allergologie gesucht}

Innerhalb der Deutschen Gesellschaft für Allergologie und klinische Immunologie (DGAKI) haben sich im März 2011 die "Junior Members" gegründet. Alle jungen Mediziner und Forscher bis zum 36. Geburtstag, die sich für das Feld der allergologischen Erkrankungen interessieren, sind herzlich eingeladen, sich zu beteiligen.

Es bietet sich die hervorragende Chance, viele Kollegen aus anderen Fachbereichen kennenzulernen, sich miteinander auszutauschen und untereinander zu vernetzen. Die DGAKI unterstützt die Junior Members und gibt ihnen die Möglichkeit, aktiv an der Gestaltung der Gesellschaft teilzunehmen. So war die erste eigene Postersession der Junior Members während des
Allergiekongresses 2011 in Wiesbaden ein voller Erfolg. Weiterhin befindet sich ein eigenes Forum im Aufbau und die Organisation von internen Workshops sowie die Bereitstellung von Informationen zu Karrierewegen in Klinik und Forschung sind geplant.

Eine Mitgliedschaft in der DGAKI ist für alle "Junior Members" kostenlos. Ebenso erhalten JM freien Zugang zum Allergo Journal und können kostenlos die jährlichen Allergiekongresse (bei aktiver Teilnahme) besuchen.

Informationen zur Mitgliedschaft und den "Junior Members" sind unter www.dgaki.de zu finden. 